TAMKANG JOURNAL OF MATHEMATICS

Volume 33, Number 1, Spring 2002

\title{
AN APPLICATION OF BRIOT-BOUQUET DIFFERENTIAL SUBORDINATION
}

\author{
JAGANNATH PATEL AND PRAVATI SAHOO
}

\begin{abstract}
By using the method of Briot-Bouquet differential subordination, we prove and sharpen some classical results in geometric function theory. We also derive some criteria for univalency for certain classes analytic functions in the open unit disc.
\end{abstract}

\section{Introduction}

Let $A_{k}(p)$ denote the class of functions of the form

$$
f(z)=z^{p}+\sum_{m=k}^{\infty} a_{p+m} z^{p+m} \quad(p, k \in N=\{1,2, \cdots\})
$$

which are analytic in the unit open disk $E=\{z:|z|<1\}$. We denote $A_{1}(p)=A(p)$. For any real number $\delta(>-p)$, we define

$$
D^{\delta+p-1} f(z)=\frac{z^{p}}{(1-z)^{\delta+p}} * f(z) \quad\left(f \in A_{k}(p) ; z \in E\right)
$$

where the symbol ' $*^{\prime}$ stands for the Hadmard product (or convolution) of two power series. We note that if $p=1$ and $\delta \in N$, then $D^{\delta} f(z)$ is the Ruscheweyh derivative [11] of $f(z)$. Further, it follows from (1.2) that for $\delta=1-p$

$$
D^{\delta+p-1} f(z)=f(z), D^{\delta+p} f(z)=(1-p) f(z)+z f^{\prime}(z),
$$

and

$$
z\left(D^{\delta+p-1} f(z)\right)^{\prime}=(\delta+p) D^{\delta+p} f(z)-\delta D^{\delta+p-1} f(z) .
$$

Let $g(z)$ and $h(z)$ be analytic in $\cdot E$. Then the function $g(z)$ is said to be subordinate to $h(z)$, written $g(z) \prec h(z)$, if $h(z)$ is univalent in $E, g(0)=h(0)$ and $g(E) \subset h(E)$.

In this paper, we propose to give some applications of Briot-Bouquet differential subordination for certain classes of analytic functions defined in terms of the operator $D^{\delta+p-1}$. Our results include several known results as special cases.

Received June 30, 2000; revised April 10, 2001.

2000 Mathematics Subject Classification. 30C45.

Key words and phrases. Differential subordination, Ruscheweyh derivative, integral transform. 


\section{Preliminaries}

Let $P_{k}$ denote the class of functions of the form

$$
p(z)=1+p_{k} z^{k}+p_{k+1} a^{k+1}+\cdots
$$

that are analytic in the open unit disk $E$.

In our present investigation, we shall require the following lemmas.

Lemma 1.([4]). Let $h(z)$ be convex univalent in $E, h(0)=1$, and $p(z) \in P_{k}$. If

$$
p(z)+\frac{z p^{\prime}(z)}{\gamma} \prec h(z)
$$

then for $\gamma \neq 0$ and $\operatorname{Re}(\gamma) \geq 0$

$$
p(z) \prec \frac{\gamma}{k} z^{-\left(\frac{\gamma}{k}\right)} \int_{0}^{z} t^{\frac{\gamma}{k}-1} h(t) d t=q(z) \prec h(z)
$$

and $q(z)$ is the best dominant.

For real or complex numbers $a, b, c(c \neq 0,-1,-2, \cdots)$, the hypergeometric function

$$
{ }_{2} F_{1}(z)={ }_{2} F_{1}(a, b ; c ; z)=1+\frac{a \cdot b}{1 . c} z+\frac{a(a+1) b(b+1)}{2 ! c(c+1)} z^{2}+\cdots
$$

represents an analytic function in $E[1, \mathrm{p} .556]$.

The following identities are well-known [1, p.556-558].

Lemma 2. For real or complex numbers $a, b$ and $c(c \neq 0,-1,-2, \cdots)$, we have

$$
\begin{aligned}
& \int_{0}^{1} t^{b-1}(1-t)^{c-b-1}(1-t z)^{-a} d t=\frac{\Gamma(b) \Gamma(c-b)}{\Gamma(c)}{ }_{2} F_{1}(a, b ; c ; z) \quad(\operatorname{Re}(c)>\operatorname{Re}(b)>0) ; \\
& { }_{2} F_{1}(a, b ; c ; z)=(1-z)^{-a}{ }_{2} F_{1}\left(a, c-b ; c ; \frac{z}{z-1}\right) ; \\
& (b+1){ }_{2} F_{1}(1, b ; b+1 ; z)=(b+1)+b z .{ }_{2} F_{1}(1, b+1 ; b+2 ; z) ; \\
& { }_{2} F_{1}(1,1 ; 2 ; z)=-z^{-1} \ln (1-z) .
\end{aligned}
$$

We shall assume throughout in the sequel that $\lambda>0, \mu>0, \delta>-p, A$ and $B$ are fixed real numbers with $A \neq B$ and $|B| \leq 1$.

\section{Main results}

We now prove

Theorem 1. For $1 \leq j \leq p$ and $f(z) \in A_{k}(p)$, let $\phi_{j}(z)$ be defined in $E$ by

$$
\phi_{j}(z)=(1-\lambda)\left(\frac{\left(D^{\delta+p-1} f(z)\right)^{(j-1)}}{z^{p-j+1}}\right)^{\mu}+\lambda\left(\frac{\left(D^{\delta+p-1} f(z)\right)^{(j)}}{z^{p-j}}\right)\left(\frac{\left(D^{\delta+p-1} f(z)\right)^{(j-1)}}{z^{p-j+1}}\right)^{\mu-1} .
$$


If

$$
\phi_{j}(z) \prec(1+\lambda(p-j))\left(\frac{p !}{(p-j+1) !}\right)^{\mu} \frac{1+A z}{1+B z}(z \in E),
$$

then

$$
\left(\frac{\left(D^{\delta+p-1} f(z)\right)^{(j-1)}}{z^{p-j+1}}\right)^{\mu} \prec\left(\frac{p !}{(p-j+1) !}\right)^{\mu} q(z)
$$

where

$$
q(z)=\left\{\begin{array}{lr}
\frac{A}{B}+\left(1-\frac{A}{B}\right)(1+B z)_{2}^{-1} F_{1}\left(1,1 ; \frac{\mu(1+\lambda(p-j))}{\lambda k}+1 ; \frac{B z}{B z+1}\right), B \neq 0 \\
1+\frac{\mu(1+\lambda(p-j))}{\mu(1+\lambda(p-j))+\lambda k} A z & , B=0
\end{array}\right.
$$

and $q(z)$ is the best dominant. Furthermore,

$$
\operatorname{Re}\left(\frac{\left(D^{\delta+p-1} f(z)\right)^{(j-1)}}{z^{p-j+1}}\right)^{\mu}>\left(\frac{p !}{(p-j+1) !}\right)^{\mu} \rho_{1},
$$

where

$$
\rho_{1}= \begin{cases}\frac{A}{B}+\left(1-\frac{A}{B}\right)(1-B)^{-1}{ }_{2} F_{1}\left(1,1 ; \frac{\mu(1+\lambda(p-j))}{\lambda k}+1 ; \frac{B}{B-1}\right), & B \neq 0 \\ 1-\frac{\mu(1+\lambda(p-j))}{\mu(1+\lambda(p-j))+\lambda k} A & , B=0 .\end{cases}
$$

The estimate in (3.5) is best possible.

Proof. Defining the function $p(z)$ in $E$ by

$$
p(z)=\left(\frac{(p-j+1) !}{p !} \cdot \frac{\left(D^{\delta+p-1} f(z)\right)^{(j-1)}}{z^{p-j+1}}\right)^{\mu} .
$$

and choosing the principal branch in (3.6), we see that $p(z) \in P_{k}$. On differentiating both the sides of (3.6) and using (3.2) in the resulting equation, we deduce that

$$
p(z)+\frac{\lambda \cdot z p^{\prime}(z)}{\mu(1+\lambda(p-j))} \prec \frac{1+A z}{1+B z} \quad(z \in E) .
$$

Since $(1+A z) /(1+B z)$ is convex (univalent) in $E$, an application of Lemma 1 yields

$$
\begin{aligned}
p(z) \prec q(z) & =\frac{\mu(1+\lambda(p-j))}{\lambda k} \cdot z^{-\frac{\mu(1+\lambda(p-j))}{\lambda k}} \int_{0}^{z} t^{\frac{\mu(1+\lambda(p-j))}{\lambda k}-1} \cdot \frac{1+A t}{1+B t} d t \\
& = \begin{cases}\frac{A}{B}+\left(1-\frac{A}{B}\right)(1+B z)^{-1}{ }_{2} F_{1}\left(1,1 ; \frac{\mu(1+\lambda(p-j))}{\lambda k}+1 ; \frac{B z}{B z+1}\right), & B \neq 0 \\
1+\frac{\mu(1+\lambda(p-j))}{\mu(1+\lambda(p-j))+\lambda k} A z & , B=0\end{cases}
\end{aligned}
$$

by change of variable followed by the use of the identities (2.2), (2.3) and (2.4). This completes the proof of (3.3). 
Next to prove (3.5), it suffices to show that

$$
\inf _{|z|<1}\{\operatorname{Re}(q(z))\}=q(-1)
$$

Indeed, we have for $|z| \leq r<1$,

$$
\operatorname{Re}\left\{\frac{1+A z}{1+B z}\right\} \geq \frac{1-A r}{1-B r}
$$

Setting

$$
g(s, z)=\frac{1+A s z}{1+B s z}, \quad(0 \leq s \leq 1, z \in E)
$$

and

$$
d \mu(s)=\frac{\mu(1+\lambda(p-j))}{\lambda k} s \frac{\mu(1+\lambda(p-j))}{\lambda k}-1 d s
$$

which is a positive measure on the closed interval $[0,1]$, we get

$$
q(z)=\int_{0}^{1} g(s, z) d \mu(s)
$$

so that

$$
\begin{aligned}
\operatorname{Re}\{q(z)\} & \geq \int_{0}^{1}\left(\frac{1-A s r}{1-B s r}\right) d \mu(s) \\
& =q(-r),|z| \leq r<1
\end{aligned}
$$

Now, letting $r \rightarrow 1^{-}$in the above inequality, we obtain the assertion (3.7).

The estimate in (3.5) is best possible as the function $q(z)$ is the best dominant.

Taking $A=1-\left(\frac{(p-j+1) !}{p !}\right)^{\mu} \cdot \frac{2 \alpha}{1+\lambda(p-j)}$ and $B=-1$ in Theorem 1, we get

Corollary 1. For $1 \leq j \leq p$ and $f(z) \in A_{k}(p)$, let $\phi_{j}(z)$ be defined in $E$ by (3.1).

(i) If

$$
\operatorname{Re}\left\{\phi_{j}(z)\right\}>\alpha \quad\left(0 \leq \alpha<(1+\lambda(p-j))\left(\frac{p !}{(p-j+1) !}\right)^{\mu}, z \in E\right)
$$

then

$$
\operatorname{Re}\left\{\frac{\left(D^{\delta+p-1} f(z)\right)^{(j-1)}}{z^{p-j+1}}\right\}^{\mu}>\rho_{2}(z \in E)
$$

where $\rho_{2}$ is given by

$$
\rho_{2}=\frac{\alpha}{1+\lambda(p-j)}+\left\{\left(\frac{p !}{(p-j+1) !}\right)^{\mu}-\frac{\alpha}{1+\lambda(p-j)}\right\} \cdot\left({ }_{2} F_{1}\left(1,1 ; \frac{\mu(1+\lambda(p-j))}{\lambda k}+1 ; \frac{1}{2}\right)-1\right) .
$$


(ii) If

$$
\operatorname{Re}\left\{\phi_{j}(z)\right\}<\alpha \quad\left(\left(1+\lambda(p-j)\left(\frac{p !}{(p-j) !}\right)^{\mu}<\alpha, z \in E\right)\right.
$$

then

$$
\operatorname{Re}\left\{\frac{\left(D^{\delta+p-1} f(z)\right)^{(j-1)}}{z^{p-j+1}}\right\}^{\mu}<\rho_{2} \quad(z \in E) .
$$

The estimates in (i) and (ii) are best possible.

Corollary 2. Let $f(z) \in A_{k}(p)$ and $2 \leq j \leq p$. If for $B \neq 0$

$$
\frac{\left(D^{\delta+p-1} f(z)\right)^{(j)}}{z^{p-j}} \prec \frac{p !}{(p-j) !} \frac{1+A^{*} z}{1+B z} \quad(z \in E)
$$

where

$$
A^{*}=\frac{B \cdot{ }_{2} F_{1}\left(1,1 ; \frac{p+k-j+1}{k} ; \frac{B}{B-1}\right)}{B+{ }_{2} F_{1}\left(1,1 ; \frac{p+k-j+1}{k} ; \frac{B}{B-1}\right)-1}
$$

then $D^{\delta+p-1} f(z)$ is p-valent in $E$.

Proof. Putting $\lambda=\mu=1$ and replacing $A$ by $A^{*}$ in Theorem 1, we deduce that

$$
\operatorname{Re}\left\{\frac{z\left(D^{\delta+p-1} f(z)\right)^{(j-1)}}{z^{p-j+2}}\right\}=\operatorname{Re}\left\{\frac{\left(D^{\delta+p-1} f(z)\right)^{(j-1)}}{z^{p-j+1}}\right\}>0 \quad(z \in E)
$$

Since $z^{p-j+2}$ is $(p-j+2)$-valently starlike in $E$, in view of Theorem 8 of Nunokawa [5], the function $D^{\delta+p-1}$ is $p$-valent in $E$.

Remarks 1. Since for $1 \leq j \leq p, \mu=\lambda=k=1$ and $\delta=1-p$,

$$
\rho_{2}>\frac{(p-j+1) !(2 \alpha+p) !}{2(p-j)+3},
$$

part (i) of Corollary 1 improves the corresponding result due to Saitoh [12].

2. Putting $j=p, k=1$ and $\delta=1-p$ in Corollary 2, we get the result contained in $[9$, Theorem 2] which inturn yields the result obtained by Nunokawa [6] for $B=-1$.

Theorem 2. Let $1 \leq j \leq p, f(z) \in A_{k}(p)$, let $\phi_{j}(z)$ be defined in $E$ by (3.1).

(i) If

$$
\operatorname{Re}\left\{\frac{\left(D^{\delta+p-1} f(z)\right)^{(j-1)}}{z^{p-j+1}}\right\}^{\mu}>\alpha\left(0 \leq \alpha<\left(\frac{p !}{(p-j+1) !}\right)^{\mu}, z \in E\right)
$$

then

$$
\operatorname{Re}\left\{\phi_{j}(z)\right\}>(1+\lambda(p-j)) \alpha \quad\left(|z|<R_{1}\right),
$$

where

$$
R_{1}=\left[\frac{\sqrt{(\lambda k)^{2}+(\mu(1+\lambda(p-j)))^{2}}-\lambda k}{\mu(1+\lambda(p-j))}\right]^{\frac{1}{k}} .
$$


(ii) If

$$
\operatorname{Re}\left\{\frac{\left(D^{\delta+p-1} f(z)\right)^{(j-1)}}{z^{p-j+1}}\right\}^{\mu}<\alpha\left(\left(\frac{p !}{(p-j+1) !}\right)^{\mu}<\alpha, z \in E\right)
$$

then

$$
\operatorname{Re}\left\{\phi_{j}(z)\right\}<(1+\lambda(p-j)) \alpha \quad\left(|z|<R_{1}\right) .
$$

The bound $R_{1}$ in (i) and (ii) is best possible.

Proof. Let us assume that (3.9) holds. Then

$$
\left(\frac{\left(D^{\delta+p-1} f(z)\right)^{(j-1)}}{z^{p-j+1}}\right)^{\mu}=\alpha+(\beta-\alpha) p(z) \quad(z \in E)
$$

where $\beta=(p ! /(p-j+1) !)^{\mu}$. Choosing the principal branch in (3.11), we see that $p(z) \in P_{k}$ and $\operatorname{Re}(p(z))>0$ in $E$. On differentiating both the sides of (3.11) and simplifying, we get

$$
\operatorname{Re}\left\{\phi_{j}(z)-(1+\lambda(p-j)) \alpha\right\}=(\beta-\alpha) \operatorname{Re}\left\{(1+\lambda(p-j)) p(z)+\frac{\lambda}{\mu} z p^{\prime}(z)\right\} .
$$

Using the well-known estimate

$$
\frac{\left|z p^{\prime}(z)\right|}{\operatorname{Re}\{p(z)\}} \leq \frac{2 k r^{k}}{1-r^{2 k}}(|z|=r<1)
$$

in (3.12), we obtain

$$
\operatorname{Re}\left\{\phi_{j}(z)-(1+\lambda(p-j)) \alpha\right\} \geq(\beta-\alpha) \operatorname{Rep}(z)\left\{(1+\lambda(p-j))-\frac{2 \lambda k r^{k}}{\mu\left(1-r^{2 k}\right)}\right\} .
$$

It is easily seen that the right-hand side of the above relation is positive when $r<R_{1}$, for $R_{1}$ is given by (3.10). Hence, $\operatorname{Re}\left\{\phi_{j}(z)\right\}>(1+\lambda(p-j)) \alpha$ for $|z|<R_{1}$.

To show that the bound $R_{1}$ is sharp, we take $f(z) \in A_{k}(p)$ defined in $E$ by

$$
\left(\frac{\left(D^{\delta+p-1} f(z)\right)^{(j-1)}}{z^{p-j+1}}\right)^{\mu}=\alpha+(\beta-\alpha)\left(\frac{1+z^{k}}{1-z^{k}}\right) \quad(z \in E)
$$

with $\beta=(p ! /(p-j+1) !)^{\mu}$. Noting that

$$
\phi_{j}(z)-(1+\lambda(p-j)) \alpha=\frac{(\beta-\alpha)}{\left(1-z^{k}\right)^{2}}\left[(1+\lambda(p-j))\left(1-z^{2 k}\right)+\frac{2 \lambda k}{\mu} z^{k}\right]=0
$$

for $z=R_{1} \exp (i \pi / k)$, we complete the proof of part (i) of the Theorem.

Similarly, we can prove part (ii) of the Theorem.

Using the same techniques as in the proof of Theorem 1, we have the following result.

Theorem 3. Let $1 \leq j \leq p, f(z) \in A_{k}(p)$ and let $\Psi_{j}(z)$ be defined in $E$ by

$$
\Psi_{j}(z)=(1-\lambda)\left(\frac{\left(D^{\delta+p-1} f(z)\right)^{(j-1)}}{z^{p-j+1}}\right)^{\mu}+\lambda \frac{\left(D^{\delta+p} f(z)\right)^{(j-1)}}{z^{p-j+1}}\left(\frac{\left(D^{\delta+p-1} f(z)\right)^{(j-1)}}{z^{p-j+1}}\right)^{\mu-1} .
$$


If

$$
\Psi_{j}(z) \prec\left(\frac{p !}{(p-j+1) !}\right)^{\mu} \frac{1+A z}{1+B z} \quad(z \in E)
$$

then

$$
\left(\frac{\left(D^{\delta+p-1} f(z)\right)^{(j-1)}}{z^{p-j+1}}\right)^{\mu} \prec \frac{p !}{(p-j+1) !} q(z)
$$

where

$$
q(z)= \begin{cases}\frac{A}{B}+\left(1-\frac{A}{B}\right)(1+B z)_{2}^{-1} F_{1}\left(1,1 ; \frac{\mu(\delta+p)}{\lambda k}+1 ; \frac{B z}{B z+1}\right), & B \neq 0 \\ 1+\frac{\mu(\delta+p)}{\mu(\delta+p)+\lambda k} A_{z} & , B=0\end{cases}
$$

and $q(z)$ is the best dominant. Furthermore,

$$
\operatorname{Re}\left(\frac{\left(D^{\delta+p-1} f(z)\right)^{(j-1)}}{z^{p-j+1}}\right)^{\mu}>\left(\frac{p !}{(p-j+1) !}\right)^{\mu} \rho_{3} \quad(z \in E)
$$

where

$$
\rho_{3}= \begin{cases}\frac{A}{B}+\left(1-\frac{A}{B}\right)(1-B)^{-1}{ }_{2} F_{1}\left(1,1 ; \frac{\mu(\delta+p)}{\lambda k}+1 ; \frac{B}{B-1}\right), & B \neq 0 \\ 1+\frac{\mu(\delta+p)}{\mu(\delta+p)+\lambda k} A & , B=0 .\end{cases}
$$

The estimate in (3.13) is best possible.

Theorem 4. For $f(z) \in A_{k}(p)$, let $F_{\lambda}(z)$ be defined in $E$ by

$$
F_{\lambda}(z)=(1-\lambda(\delta+1)) D^{\delta+p-1} f(z)+\lambda(\delta+p) D^{\delta+p} f(z) \quad(0 \leq \lambda, z \in E) .
$$

If

$$
\frac{F_{\lambda}^{(j)}(z)}{z^{p-j}} \prec(1-\lambda+\lambda p) \frac{p !}{(p-j) !} \frac{1+A z}{1+B z} \quad(z \in E)
$$

for $0 \leq j \leq p$, then

$$
\frac{\left(D^{\delta+p-1} f(z)\right)^{(j)}}{z^{p-j}} \prec \frac{p !}{(p-j) !} q(z),
$$

where

$$
q(z)= \begin{cases}\frac{A}{B}+\left(1-\frac{A}{B}\right)(1+B z)^{-1}{ }_{2} F_{1}\left(1,1 ; \frac{1-\lambda+\lambda p}{\lambda k}+1 ; \frac{B z}{B z+1}\right), & B \neq 0 \\ 1+\frac{1-\lambda+\lambda p}{1-\lambda+\lambda(p+k)} A z & , B=0\end{cases}
$$

and $q(z)$ is the best dominant. Further, for $0 \leq j \leq p$ we have

$$
\operatorname{Re}\left\{\frac{\left(D^{\delta+p-1} f(z)\right)^{(j)}}{z^{p-j}}\right\}>\frac{p !}{(p-j) !} \rho_{4} \quad(z \in E),
$$


where $\rho_{4}$ is given by

$$
\rho_{4}= \begin{cases}\frac{A}{B}+\left(1-\frac{A}{B}\right)(1-B)_{2}^{-1} F_{1}\left(1,1 ; \frac{1-\lambda+\lambda p}{\lambda k}+1 ; \frac{B}{B-1}\right), & B \neq 0 \\ 1-\frac{1-\lambda+\lambda p}{1-\lambda+\lambda(p+k)} A & , B=0 .\end{cases}
$$

The estimate in (3.16) is best possible.

Proof. Using (3.14) and the identity (1.3), it follows that

$$
F_{\lambda}^{(j)}(z)=(1-\lambda+\lambda j)\left(D^{\delta+p-1} f(z)\right)^{(j)}+\lambda z\left(D^{\delta+p-1} f(z)\right)^{(j+1)}
$$

for $0 \leq j \leq p$. Letting

$$
p(z)=\frac{(p-j) !}{p !} \frac{\left(D^{\delta+p-1} f(z)\right)^{(i)}}{z^{p-j}} \quad(z \in E),
$$

we note that $p(z) \in P_{k}$. On differentiating both the sides of (3.18), using (3.15) and (3.17) in the resulting equation followed by a simple calculation, we obtain

$$
p(z)+\frac{\lambda}{1-\lambda+\lambda p} z p^{\prime}(z) \prec \frac{1+A z}{1+B z} \quad(z \in E) .
$$

The remaining part of the proof is similar to that of Theorem 1. So we omit the details.

Setting $\delta=1-p, k=1, A=1-\frac{2 \alpha(p-j) !}{p !(1-\lambda+\lambda p)}$ and $B=-1$ in Theorem 4, we obtain the following.

Corollary 3. Let $0 \leq j \leq p, f(z) \in A_{p}$ and $F_{\lambda}(z)=(1-\lambda) f(z)+\lambda z f^{\prime}(z)$ for $\lambda \geq 0$.

(i) If

$$
\operatorname{Re}\left\{\frac{F_{\lambda}^{(i)}(z)}{z^{p-j}}\right\}>\alpha \quad\left(0 \leq \alpha<\frac{p !(1-\lambda+\lambda p)}{(p-j) !}, z \in E\right)
$$

then

$$
\operatorname{Re}\left\{\frac{f^{(j)}(z)}{z^{p-j}}\right\}>\frac{\alpha}{1-\lambda+\lambda p}+\left\{\frac{p !}{(p-j) !}-\frac{\alpha}{1-\lambda+\lambda p}\right\} \cdot\left({ }_{2} F_{1}\left(1,1 ; \frac{1+\lambda p}{\lambda}+1 ; \frac{1}{2}\right)-1\right) .
$$

(ii) If

$$
\operatorname{Re}\left\{\frac{F_{\lambda}^{(j)}(z)}{z^{p-j}}\right\}<\alpha \quad\left(\frac{p !(1-\lambda+\lambda p)}{(p-j) !}<\alpha, z \in E\right)
$$

then

$$
\operatorname{Re}\left\{\frac{f^{(j)}(z)}{z^{p-j}}\right\}<\frac{\alpha}{1-\lambda+\lambda p}+\left\{\frac{\alpha}{1-\lambda+\lambda p}-\frac{p !}{(p-j) !}\right\} \cdot\left({ }_{2} F_{1}\left(1,1 ; \frac{1+\lambda p}{\lambda}+1 ; \frac{1}{2}\right)-1\right) .
$$


The estimates in $(i)$ and $(i i)$ are best possible.

Remark. We note that the results contained in Theorem 4 and Corollary 3 improves the corresponding work of Yang [13].

Theorem 5. Let $0 \leq j \leq p, f(z) \in A_{k}(p)$ and $F_{\lambda}(z)$ be defined in $E$ by (3.14).

(i) If

$$
\operatorname{Re}\left\{\frac{\left(D^{\delta+p-1} f(z)\right)^{(j)}}{z^{p-j}}\right\}>\alpha \quad\left(0 \leq \alpha<\frac{p !}{(p-j) !}, z \in E\right)
$$

then

$$
\operatorname{Re}\left\{\frac{F_{\lambda}^{(j)}(z)}{z^{p-j}}\right\}>(1-\lambda+\lambda p) \alpha \quad\left(|z|<R_{2}\right)
$$

where

$$
R_{2}=\left[\frac{\sqrt{(\lambda k)^{2}+(1-\lambda+\lambda p)^{2}}-\lambda k}{(1-\lambda+\lambda p)}\right]^{\frac{1}{k}} .
$$

(ii) If

$$
\operatorname{Re}\left\{\frac{\left(D^{\delta+p-1} f(z)\right)^{(j)}}{z^{p-j}}\right\}<\alpha \quad\left(\frac{p !}{(p-j) !}<\alpha, z \in E\right)
$$

then

$$
\operatorname{Re}\left\{\frac{F_{\lambda}^{(j)}(z)}{z^{p-j}}\right\}<(1-\lambda+\lambda p) \alpha \quad\left(|z|<R_{2}\right)
$$

The bound $R_{2}$ in $(i)$ and $(i i)$ is best possible.

Proof. Defining the function $p(z)$ in $E$ by

$$
\frac{\left(D^{\delta+p-1} f(z)\right)^{(j)}}{z^{p-j}}=\alpha+(\beta-\alpha) p(z)
$$

with $\beta=p ! /(p-j)$ !, we see that $p(z) \in P_{k}$ and $\operatorname{Re}(p(z))>0$ in $E$. Differentiating both the sides of (3.19) and using (3.17) in the resulting equation, we obtain

$$
\operatorname{Re}\left\{\frac{F_{\lambda}^{(j)}(z)}{z^{p-j}}-(1-\lambda+\lambda p) \alpha\right\}=(\beta-\alpha) \operatorname{Re}\left\{(1-\lambda+\lambda p) p(z)+\lambda z p^{\prime}(z)\right\} .
$$

The estimates in (i) and (ii) can now be deduced on the same lines as that of Theorem 2.

The bound $R_{2}$ can be seen to be sharp by taking $f \in A_{k}(p)$ defined by

$$
\frac{\left(D^{\delta+p-1} f(z)\right)^{(j)}}{z^{p-j}}=\alpha+(\beta-\alpha) \frac{1+z^{k}}{1-z^{k}} \quad(0 \leq j \leq p, z \in E) .
$$

Remark. We note that our method of proof of Theorem 5 is much simpler than that of Yang [13].

We now study certain integral transforms of functions in $A_{k}(p)$. 
Theorem 6. For $f(z) \in A_{k}(p)$, let $I_{\gamma}(z)$ be defined in $E$ by

$$
I_{\gamma}(z)=\frac{\gamma+p}{z^{\gamma}} \int_{0}^{z} t^{\gamma-1} f(t) d t \quad(\gamma>-p)
$$

If

$$
\frac{\left(D^{\delta+p-1} f(z)\right)^{(j)}}{z^{p-j}} \prec \frac{p !}{(p-j) !} \cdot \frac{1+A z}{1+B z} \quad(0 \leq j \leq p, z \in E)
$$

then

$$
\frac{\left(D^{\delta+p-1} I_{\gamma}(z)\right)^{(j)}}{z^{p-j}} \prec \frac{p !}{(p-j) !} q(z),
$$

where

$$
q(z)=\left\{\begin{array}{lr}
\frac{A}{B}+\left(1-\frac{A}{B}\right)(1+B z)^{-1} F_{1}\left(1,1 ; \frac{\gamma+p}{k}+1 ; \frac{B z}{B z+1}\right), & B \neq 0 \\
1+\frac{\gamma+p}{\gamma+p+k} A z & , B=0
\end{array}\right.
$$

and $q(z)$ is the best dominant. Furthermore, for $0 \leq j \leq p$ we have

$$
\operatorname{Re}\left\{\frac{\left(D^{\delta+p-1} I_{\gamma}(z)\right)^{(j)}}{z^{p-j}}\right\}>\frac{p !}{(p-j) !} \rho_{5} \quad(z \in E),
$$

where $\rho_{5}$ is given by

$$
\rho_{5}= \begin{cases}\frac{A}{B}+\left(1-\frac{A}{B}\right)(1-B)_{2}^{-1} F_{1}\left(1,1 ; \frac{\gamma+p}{k}+1 ; \frac{B}{B-1}\right), & B \neq 0 \\ 1-\frac{\gamma+p}{\gamma+p+k} A & , B=0 .\end{cases}
$$

The estimate in (3.23) is best possible.

Proof. Letting

$$
p(z)=\frac{(p-j) !}{p !} \frac{\left(D^{\delta+p-1} I_{\gamma}(z)\right)^{(j)}}{z^{p-j}} \quad(z \in E),
$$

we see that $p(z) \in P_{k}$. On differentiating the above expression, using (3.21) and the identity

$$
z\left(D^{\delta+p-1} I_{\gamma}(z)\right)^{(j+1)}=(\gamma+p)\left(D^{\delta+p-1} f(z)\right)^{(j)}-(\gamma+j)\left(D^{\delta+p-1} I_{\gamma}(z)\right)^{(j)}
$$

in the resulting equation, we get

$$
p(z)+\frac{z p^{\prime}(z)}{\gamma+p} \prec \frac{1+A z}{1+B z} \quad(z \in E) .
$$

Now, the assertion (3.22) and the estimate (3.23) follow by employing the techniques that proved Theorem 1.

Taking $A=1-2 \alpha \frac{(p-j) !}{p !}$ and $B=-1$ in Theorem 6 , we get

Corollary 4. Let $0 \leq j \leq p, f(z) \in A_{k}(p)$ and $I_{\gamma}(z)$ be defined in $E$ by (3.20). 
(i) If

$$
\operatorname{Re}\left\{\frac{\left(D^{\delta+p-1} f(z)\right)^{(j)}}{z^{p-j}}\right\}>\alpha \quad(z \in E),
$$

then

$$
\operatorname{Re}\left\{\frac{\left(D^{\delta+p-1} I_{\gamma}(z)\right)^{(j)}}{z^{p-j}}\right\}>\alpha+\left\{\frac{p !}{(p-j) !}-\alpha\right\} \cdot\left({ }_{2} F_{1}\left(1,1 ; \frac{\gamma+p}{k}+1 ; \frac{1}{2}\right)-1\right) \quad(z \in E) .
$$

(ii) If

$$
\operatorname{Re}\left\{\frac{\left(D^{\delta+p-1} f(z)\right)^{(j)}}{z^{p-j}}\right\}<\alpha \quad\left(\frac{p !}{(p-j) !}<\alpha, z \in E\right),
$$

then

$$
\operatorname{Re}\left\{\frac{\left(D^{\delta+p-1} I_{\gamma}(z)\right)^{(j)}}{z^{p-j}}\right\}<\alpha-\left\{\alpha-\frac{p !}{(p-j) !}\right\} \cdot\left({ }_{2} F_{1}\left(1,1 ; \frac{\gamma+p}{k}+1 ; \frac{1}{2}\right)-1\right) \quad(z \in E) .
$$

The estimates in ( $i)$ and (ii) are best possible.

Following the lines of proof of Theorem 2, we have

Theorem 7. Let $0 \leq j \leq p$ and $I_{\gamma}(z)$ be defined by (3.20).

(i) If

$$
\operatorname{Re}\left\{\frac{\left(D^{\delta+p-1} I_{\gamma}(z)\right)^{(j)}}{z^{p-j}}\right\}>\alpha \quad\left(0 \leq \alpha<\frac{p !}{(p-j) !}, z \in E\right),
$$

then

$$
\operatorname{Re}\left\{\frac{\left(D^{\delta+p-1} f(z)\right)^{(j)}}{z^{p-j}}\right\}>\alpha \quad\left(|z|<R_{3}\right)
$$

where

$$
R_{3}=\left[\sqrt{(\gamma+p)^{2}+k^{2}}+k\right]^{\frac{-1}{k}}
$$

(ii) If

$$
\operatorname{Re}\left\{\frac{\left(D^{\delta+p-1} I_{\gamma}(z)\right)^{(j)}}{z^{p-j}}\right\}<\alpha \quad\left(\frac{p !}{(p-j) !}<\alpha, z \in E\right),
$$

then

$$
\operatorname{Re}\left\{\frac{\left(D^{\delta+p-1} I_{\gamma}((z))^{(j)}\right.}{z^{p-j}}\right\}<\alpha \quad\left(|z|<R_{3}\right)
$$

The bound $R_{3}$ in $(i)$ and (ii) are best possible.

\section{Acknowledgements}

The authors are thankful to the referee for his helpful comments which improves the presentation of the paper. 


\section{References}

[1] M. Abramowitz and I. A. Stegun, Hand Book of Mathematical Functions, Dover Publications, Inc., New York, 1971.

[2] S. D. Bernardi, Convex and starlike univalent functions, Trans. Amer. Math. Soc., 135(1969), 429-446.

[3] R. J. Libera, Some classes of regular univalent functions, Proc. Amer. Math. Soc., 16(1965), 755-758.

[4] S. S. Miller and P. T. Mocanu, Differential subordinations and univalent functions, Michigan Math. J., 28(1981), 157-171.

[5] M. Nunokawa, On the theory of multivalent functions, Tsukuba J. Math., 11(1987), 273-286.

[6] M. Nunokawa, Differential inequalities and Caratheodory function, Proc. Japan Acad., 65 Ser. A (1989), 326-328.

[7] M. Obradovic, A property of the class of functions whose derivative has a positive real part, Publications De L' Institute Mathematique, Nouvelle Serie, tome, 45(59) (1989), 81-84.

[8] S. Owa, M. Obradovic and M. Nunokawa, On certain analytic functions and subordinations, Appl. Math. Letters, 3(1990), 41-45.

[9] J. Patel and S. Rout, An application of differential subordination, Rend. di Mat. Serie VII, 14(1994), 367-384.

[10] S. Ponnusamy and O. P. Juneja, Some applications to Briot-Bouquet differential subordinations, Ann. Univ., Mariae Curiesklodowska, XLII(1988), 129-144.

[11] S. Ruscheweyh, New criteria for univalent functions, Proc. Amer. Math. Soc., 49(1975), 109-115.

[12] H. Saitoh, Properties of certain analytic functions, Proc. Japan Acad., 65, Serie A. (1989), 131-134.

[13] D. Yang, Some properties of certain analytic functions, Soochow J. Math., 23 (1997), 63-70.

Department of Mathematics, Utkal University, Vani Vihar Bhubaneswar-751004, India. 